\title{
Stochastic Resonance: from climate to biology
}

\author{
Roberto Benzi ${ }^{1}$ \\ ${ }^{1}$ Dipartimento di Fisica and INFN, Università di Roma "Tor Vergata", \\ Via della Ricerca Scientifica 1, 00133 Roma, Italy.
}

(Dated: October 1, 2018)

\begin{abstract}
In this paper I will review some basic aspects of the mechanism of stochastic resonance. Stochastic resonance was first introduced as a possible mechanism to explain long term climatic variation. Since then, there have been many applications of stochastic resonance in physical and biological systems. I will show that in complex system, stochastic resonance can substantially change as a function of the "system complexity". Also, I will briefly mention how to apply stochastic resonance for the case of Brownian motors.
\end{abstract}

PACS numbers:

\section{INTRODUCTION}

The first numerical simulation providing strong evidence of Stochastic Resonance was performed by Angelo Vulpiani and myself in a rather exciting night on February 1980. Together with Alfonso Sutera and Giorgio Parisi, we were trying, at that time, to understand whether a relatively small periodic forcing can be amplified by internal non linear stochastic dynamics, leading to a possible understanding of the 100 Kyear cycle observed in climate records. The first version of our paper was not accepted for publication in Journal of Geophyisical Research and in Journal of Atmospheric Science. Eventually the paper was published in Tellus in 1982 1 together with a similar paper by Nicolis and Nicolis [2] who, independently, were proposing the same mechanism for climatic change. Short after that february night in 1980, we were able to provide a quite general understanding how the mechanism works and how to generalize it for chaotic systems. Both theory and generalization appeared as a letter in J. Phys A in 1981 [3], i.e. one year before the Tellus paper!

We were, and still we are, convinced that Stochastic Resonance is a rather new and conceptually important phenomenon in science. For almost a decade, only few colleagues were shearing with us the same feeling. Eventually, after the paper of Moss et.al. [4] and some new theoretical work [5] stochastic resonance became a fashionable and interesting research topic in many different scientific areas, see [6] for a review.

Resonance is a kind of magic word in physics and there is no surprise that a new resonance mechanism can excite the scientific community. In our case, the name stochastic resonance was introduce because of a short discussion with J.Imbrie on march 1980. Just few days after our first numerical simulation, I participated to a climate meeting in Erice where I gave a short talk on our results. J.Imbrie, one of the most famous scientist working on paleoclimate, asked whether what we found was somehow similar to a resonance and my answer was: "not exactly! It is a kind of stochastic resonance!". This is how the name was in- troduced in literature. In some sense, we can think of a "resonance" as follows. In the standard resonance mechanism, think for instance of a damped harmonic oscillator, the amplification of the external forcing can be related, mathematically, to a singularity in the complex plane of the green function of the problem. The real part of the singularity is, of course, the resonance frequency. In chaotic or stochastic systems, there are singularities in the complex plane but on the imaginary axis. The mechanism of stochastic resonance provides a way to shift the singularity on the complex plane with a non zero real part. This rather cumbersome view of stochastic resonance may be not entirely clear but it tries to justify why the word "resonance" can still be used beside any historical reason.

In this paper, I will review the main idea on stochastic resonance with an emphasis on climate research, where it was originally proposed. I will also show how stochastic resonance is still an interesting subject to work on and in particular I will discuss some new findings in simple and complex systems. Finally, I will mention how mechanisms related to stochastic resonance are of interest in biology and in the wide research field of brownian motors.

\section{THE MECHANISM OF STOCHASTIC RESONANCE IN CLIMATE THEORY}

Climate is one of the most complex system in nature and it is a major scientific challenge to understand the basic mechanisms leading to climate changes. In the past, climate shows quite remarkable changes over all times scales. In particular, one of the most striking aspects of past climate changes is the so called $100 \mathrm{ky}$ cycles observed in paleoclimatic records. The cycle itself is not at all periodic showing sudden warming phases followed by gentle decreases of temperature. This saw-tooth behaviour is in phase with the so called Milankovitch cycle, i.e. to the change of global radiation of the Sun due to astronomical change in Earth orbit. This is the only global (i.e. averaged) change experienced in the incoming radi- 


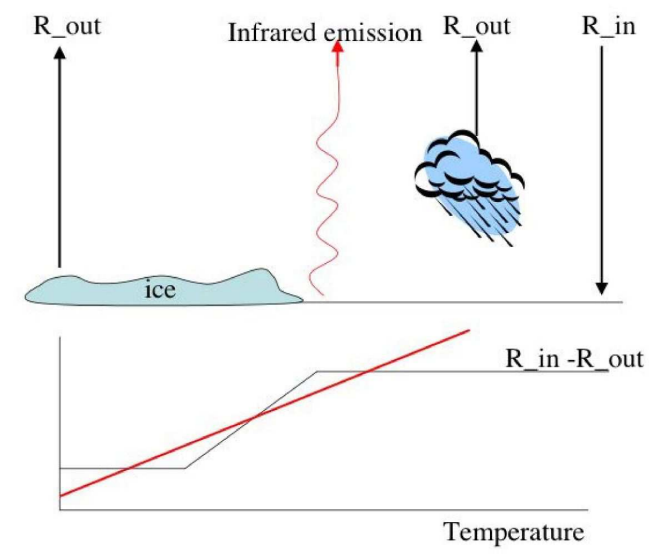

FIG. 1: The energy balance climate model. In the upper layer we show the basic variables of the model: the incoming radiation $R_{\text {in }}$ and the out coming radiation $R_{\text {out }}$ and the infrared emission. In the lower panel we show the albedo as a function of temperature $R_{\text {in }}-R_{\text {out }}=(1-\alpha(T)) R_{\text {in }}$. The thick line represents the infrared emission.

ation. Is that possible to relate the astronomical forcing to the observed climate change? This is the basic question which puzzles scientific research since many decades. A simple computation shows that it is not easy to guess the correct answer.

Let us consider the simplest possible climate model. We consider the averaged Earth temperature $T$ as the basic variable we want to describe, the precise meaning of average is of marginal interest for the time being. We know how much radiation is incoming, let us say $R_{i n}$. The out coming radiation $R_{\text {out }}$ depends on the earth's temperature $T$ (infrared emission) and reflection from the surface, which can be written as $\alpha R_{i n}$. Assuming $C_{E}$ the earth's thermal inertia, we can write the energy balance model as:

$$
C_{E} \frac{d T}{d t}=R_{\text {in }}-R_{\text {out }}
$$

where $R_{\text {out }}=\alpha R_{\text {in }}+E_{I}, E_{I}$ being the infrared emission.

What we might call Earth'climate is just the stationary solution of equation (1). While $R_{i n}$ is independent on $T$, both $\alpha$ and $E_{I}$ should be dependent on the Earth's climate which, in our simple case, is described by the averaged temperature $T$. In a first approximation, we can think that $E_{I}$ is linearly dependent on $T$ (a more complex behaviour does not substantially change the results). The so called albedo $\alpha$ is also a $T$ dependent quantity. More specifically, we expect $\alpha$ to be quite large for ice regions. An appropriate way to think of the function $\alpha(T)$ is shown in figure (11). Following the cartoon shown in the same figure, it is clear that there exist more than one stationary solution of equation (10), corresponding to three different possible Earth climates. The coldest of the possible climates is referred to as "ice cover earth" climate, i.e. the case where almost all incoming radiation is reflected back in the space. A careful estimate of the albedo effect and the infrared radiation, obtained by current observations, shows that the warmest possible climate is close to the current Earth climate (we discard the intermediate climate simply because it is dynamically unstable). Thus equation (11) gives us a quite amazing result as it explains our present climate state. Note that an increase of $\mathrm{CO}_{2}$ in the atmosphere is equivalent, in our simple model, to a change in $E_{I}$ represented by the thick line in the lower panel of figure (11).

Our simple model tells us that there is an interesting feature in climate dynamics represented by the instability of the intermediate climate state. What is the physical origin of this instability? The answer is related to the complex mechanism of cloud formation. Clouds by themselves reflect incoming radiation. When the temperature increases, the albedo start to increases as well because more clouds are expected to form. Eventually, for large enough temperature, the amount of cloud formation is balanced by precipitation leading to a constant value of $\alpha$. For the time being, let us concentrate on the present (stable) climate. The orbital forcing, discovered by Milankovitch, is a small amplitude modulation of $R_{i n}$ in time, i.e.

$$
R_{\text {in }}(t)=R_{\text {in }}^{S}+A \cos (\omega t)
$$

Let us consider the effect of the Milankovitch forcing in equation (1). If $T$ is always quite close to the stationary (present) climate $T_{0}$, we can estimate $\delta T \equiv T-T_{0}$ as:

$$
\frac{d \delta T}{d t}=-\frac{1}{\tau} \delta T+A \cos (\omega t)
$$

where $1 / \tau$ is the relaxation time of the present climate. More precisely $\tau$ can be estimated by the equation:

$$
\frac{1}{\tau}=-\left.\frac{1}{C_{E}} \frac{\left[E_{I}-(1-\alpha(T)) R_{i n}\right]}{d T}\right|_{T=T_{0}}
$$

After an appropriate estimate of $A$, one can easily show using (3) that the effect of the orbital forcing on the present climate is of order $A \tau \sim 0.5 K$, much smaller than the $10 K$ observed in paleoclimatic records. Thus, it seems that orbital forcing can hardly explain the glacial/interglacial transition experienced by the Earth climate.

In order to make progress we need to supply our simple model by new features. An important feature is to assume that there exist more than one stable climate state close to our present value $T_{0}$. More precisely, we shall assume that the albedo feedback mechanim (described in terms of clouds and ice) can eventually lead to a more complex behavior near our present climate leading to two stable climate states $T_{1}$ and $T_{0}$ separated by about $10 \mathrm{~K}$ see figure (2). This is a rather ad hoc assumption which 
is, at any rate, consistent with observations on albedo. Still, this feature does not really change our conclusion since the orbital forcing is too small to provide a suitable mechanism to produce transitions between $T_{0}$ and $T_{1}$.

The situation can improve dramatically if we consider the following three non trivial statements:

- Many complex systems can be described by means of slow variables and fast variables, even if there is no explicit time scale separation between the two kind of variables. Fast variables may be considered as "noise" acting on the dynamics of slow variables.

- For Earth climate, the averaged temperature in (11) should be considered as a slow variable with respect to the fast variables due to weather variability.

- When there exist multiple equilibriums, the noise can introduce a long (random) time scale to switch from one equilibrium to another.

The three statements are no trivial and should be explained carefully. For many decades, the splitting among slow and fast variables have been considered a well known feature of molecular motion. At the atomic scale, particles continuously experience short time interactions with other atoms while the hydrodynamic behavior (the slow variables) is described by a suitable space average over the molecular chaos. Looking at the fluctuating properties of a turbulent flow, there exists a well defined scale separation (both in time and in space) from molecular motion and hydrodynamic flow. Nevertheless, even in a turbulent flow, i.e. disregarding any molecular motion, we can still distinguish large and small scale fluctuations. In this case, however, there is no spectral gap identifying any scale separation among scales. Therefore, one may be tempted to state that, in this case, the concepts of fast and slow variables are poorly defined. It turns out that such a statement is too limited. There exist experimental systems where the large scale motion can be thought as the slow variable superimposed to a noise (turbulent) small scale background acting on the system. Recently [7], it has been shown that the above picture can explain qualitatively and quantitatively the numerical results observed in a simplified model of turbulence. Thus, in most complex systems the separation of large scale, slow variables and small scales, fast variables does correctly picture the dynamics in a self consistent way.

If we apply the above discussion on the Earth climate (second item), then on the time scale corresponding to the orbital forcing, the day by day weather fluctuations should be considered as noise (fast) variables acting on the climate system. This is not just a way of thinking. Disregarding the effect of noise is a major limitation for a correct description of the physical properties of climate. It took a long time, and it is still on going, for the scientific community to accept that noise is not a measure of our "ignorance" but just a physical feature of most complex systems.

The third item is more delicate and needs to be explained in details. Let us assume that our climate system (although the discussion is true for any system) shows two stable equilibriums whose temperature difference is $2 \Delta T$. In this case, it is well known that the effect of the noise can induce transitions between the equilibria. The characteristic time $\tau_{L}$ for the transition can be estimated as:

$$
\tau_{L} \sim \tau \exp \left(\Delta T^{2} / \sigma \tau\right)
$$

where as in equation (3) $\tau$ is defined as the (fast) relaxation time over one of the stable equilibriium and $\sigma$ is the variance of the noise (weather fluctuations) acting on the system. Thus our year by year temperature is assumed to be decomposed in the climatic component $T$ and a fast noise component. In equation (4), $\tau_{L}$ should be considered an average time to switch from one equilibrium to another. In other words, the transition time between the two equilibriums $\tau_{e q}$ is a random variable with average value $\tau_{L}$. Actually, under some quite generic conditions, we can predict the probability distribution of $\tau_{e q}$ which is

$$
P\left(\tau_{e q}\right)=\frac{\exp \left(-\tau_{e q} / \tau_{L}\right)}{\tau_{L}}
$$

Let me summarize the new feature we have introduced in the simple energy balance model (1). We have assumed a new form of the albedo which is providing three different climate states around $T_{0}$, let us call them $T_{1}$ (stable), $T_{i}$ (unstable) and $T_{0}$. The shape of the new albedo is shown in figure (2). We assume that $T_{0}-T_{1}=10 \mathrm{~K}$ and $T_{0}-T_{i}=T_{i}-T_{1}$. Next we have introduced an external noise which takes into account the dynamics of the fast variables, whatever they are, not represented by the slow component $T$. We can rescale $T$ near $T_{i}$ and simplify the equations by using $T=T_{i}+\Delta T X$ with $\Delta T=5^{\circ} \mathrm{K}$ :

$$
\frac{d X}{d t}=X-X^{3}+\sqrt{\sigma} \eta(t)
$$

where $\eta(t)$ is a gaussian random variable with unit variance $\left(\left\langle\eta(t) \eta\left(t^{\prime}\right)\right\rangle=\delta\left(t-t^{\prime}\right)\right)$ and $\sigma$ is the noise strength. Notice that we have also rescaled the time unit. In order to do some computation what we still need is the strength of the noise. We can obtain some information by the following trick. In our present climate (i.e. $T_{0}$ ) we have observational data which tells us about average temperature fluctuation. Also, we know from General Circulation Models, which is the time correlation for temperature fluctuations. Thus, we can estimate the strength of the noise by linearizing our climate model around $T_{0}$ and using the fluctuation-dissipation theorem, i.e. temperature variance $=$ noise strength $/$ (2 relaxation time $)$. Once we know the noise strength $\sigma$ we can compute the average 


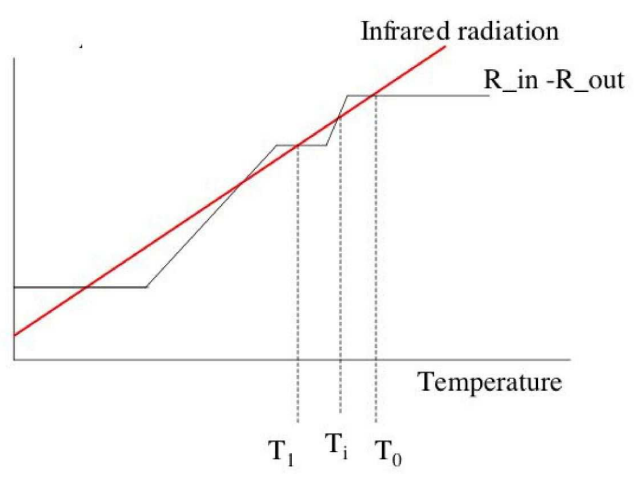

FIG. 2: The albedo feedback for an energy balance model with three climate states near $T_{0}$. The model is conceptually similar to the one described in figure (1) with a change in the albedo.

switching time from $T_{0}$ to $T_{1}$. It turns out that, by using (44) the average transition time between the two stable states of our climate model is close to 50,000 years! This is a rather surprising and interesting result because it tells us that there exists a rather long time scale $(50,000$ years) introduced by the nonlinearity in the model and the noise, to be compared against the quite short time scale of the model, namely the deterministic relaxation time towards one of the two stable equilibriums which is of the order of 10 years. However, this is not enough because the characteristic time of 50,000 years is a random time with an exponential probability distribution. Therefore nonlinearity and noise are not enough to get a periodic behavior of the temperature.

In order to get a periodic behavior, the idea is to introduce the Milankovitch effect in the system. After a simple computation one gets the following results:

$$
\frac{d X}{d t}=X-X^{3}+A \sin (\omega t)+\sqrt{\sigma} \eta(t)
$$

An appropriate rescaling in terms of physical quantities gives us the value of $A \sim 0.11$ for the Milankovitch terms and $\omega=2 \pi / 10^{5} \times 1 / 10$. Without the noise, the value of $X$ changes periodically in time with an amplitude order $A$. Going back to the temperature, this implies that, without noise, we have a periodic behavior of temperature with amplitude $A \Delta T \sim 0.5 \mathrm{~K}$. The situation changes completely when we consider the effect of the noise. A correct understanding of the noise effect can be achieved by considering the simplified equation:

$$
\frac{d X}{d t}=X-X^{3}+A \sqrt{\sigma} \eta(t)
$$

i.e. without any time dependency on the external forcing. It is crucial to remind that for any stochastic differential equation of the form:

$$
\frac{d X}{d t}=-\frac{\partial V}{\partial X}+\sqrt{\sigma} \eta(t)
$$

a

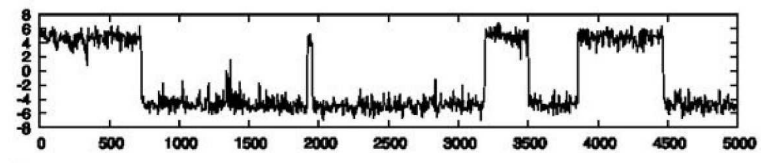

b

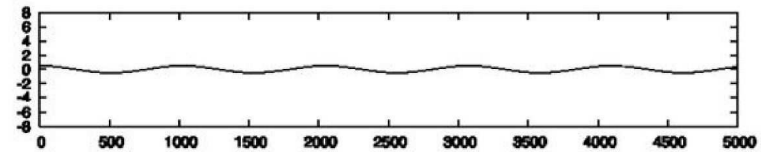

c

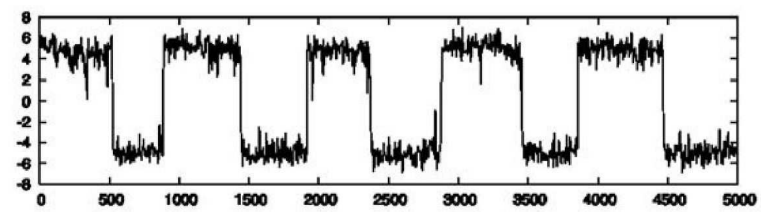

FIG. 3: Numerical simulation of equation (77) in three different cases. In upper panel, we show the solution with the noise and $A=0$. In the middle panel we show the perturbing forcing $A \cos (2 \pi \omega t)$ while in the lower panel we show the solution with $A \neq 0$. The effect of the small periodic forcing is to synchronized the random switching from one climate states to the other, i.e. to get a stochastic resonance.

the "equilibrium" probability distribution $P(X)$ of $X$ is given by:

$$
P(X)=Z_{N} \exp (-2 V(X) / \sigma)
$$

where $Z_{N}$ is a normalization factor. In our case $V(X)=$ $-1 / 2 X^{2}+1 / 4 X^{4}-A X(8)$ and we obtain from (10)

$$
P(X ; A)=P(X ; A=0) \exp (2 A X / \sigma)
$$

The probability distribution when $A \neq 0$ changes dramatically the probability distribution when $A=0$. Even for small $A$ the exponential factor $\exp (2 A / \sigma)$ can be rather large, i.e. order 30 in our case. The consequence of this simple calculation is that the probability to be near $X \sim 1$ is 30 times larger than to be in $X=-1$. Now it is quite clear that if $A$ is slowly varying as $\cos (\omega t)$ in time than the probability distribution is peaked periodically in \pm 1 with almost the same period of $2 \pi / \omega$. This is the very essence of stochastic resonance.

In figure (3) we show the numerical simulation of (7) and in particular the upper panel is $X(t)$ for $A=0$, the pnnel in the center shows the behaviour of $A(t)$ while the lower panel is the solution of (77). As we can see, the effect of the small periodic perturbation is to synchronize the "random" behavior of $X$ and to produce an almost periodic output. In some sense, stochastic resonance is a counter intuitive phenomenon because without noise the system shows a small amplitude modulation around one of the two stable steady states \pm 1 , while adding the noise we obtain a large amplitude effect with the same period.

One has to be a little bit carefully in understanding the possible results of (7). As we said, the modulation of $A$ in 
time should be slow enough in order to get a stochastic resonance. We can be more quantitative using the following simple argument. Let us go back to equation (8). We can compute the probability distribution of the random time to go from +1 to -1 and we already know that the probability distribution is exponential. Let $\tau_{1}(+1)$ the average exit time, then the variance of the random switching time is also $\tau_{1}(+1)$. Finally we know how to relate $\tau_{1}(+1)$ to $A$, namely by using the expression:

$$
\tau_{1}(+1) \sim \exp \left(\frac{2}{\sigma}\left(\Delta V_{0}+A\right)\right)
$$

where in our case $\Delta V_{0} \equiv 1 / 4$. Thus the requirement we need is that $\tau_{1}(+1) \ll 2 \pi / \omega$ and $\tau_{1}(-1) \gg 2 \pi / \omega$ where $\tau_{1}(-1)$ is the average exit time starting with the initial condition $X=-1$. The two requirements implies

$$
\begin{aligned}
\exp \left(2 \frac{\Delta V_{0}}{\sigma}\right) & \sim \frac{2 \pi}{\omega} \\
\exp \left(\frac{2}{\sigma}\left(\Delta V_{0}-A\right)\right) & \ll \frac{2 \pi}{\omega}
\end{aligned}
$$

The meaning of the above inequalities is represented in the cartoon of figure (44). The time is in the counterclockwise starting with the left top panel. We start at $X=1$ and as time goes by, the system at $t=T / 2$ has a very high probability to jump in $X=-1$ if (14) is satisfied. Then at $t=T$ we have the same effect for the transition $X=-1 \rightarrow X=1$. The two conditions (13)14) tell us that if the noise is too small then we will never see anything and, on the other hand, if the noise is too large transitions will happen regardless of the external periodic forcing $A$. Thus, there is a range of $\sigma$ (i.e. the resonant range) where we obtain periodic transitions in phase with the external forcing.

One can highlight the resonance effect also by using another kind of variable. Let $X(t)$ the solution of (7) and let us denote $F_{X}(\nu)$ its Fourier transform. Then $\left|F_{X}(\nu)\right|^{2}$ represents the power spectrum of $X$. We should expect that $\left|F_{X}(\nu=\omega)\right|^{2}$ is a function of the noise with some maximum value for the "resonant noise" $\sigma_{R}$. This is indeed the case as shown in figure (5) , where $\mid F_{X}(\nu=$ $\omega)\left.\right|^{2}$ is plotted against the $\sigma$, while in the insert we show $\left|F_{X}(\nu)\right|^{2}$ for $\sigma=\sigma_{R}$.

Let me know go back to the climate. As we have seen, the effect of a small periodic forcing in our "simplified" climate model is to produce a periodic glacial to interglacial transition in phase with the Milankovitch forcing. This is a quite good new. However, the signal shown in figure (3) poorly compares against the observed behavior of the "proxy" earth temperature. The latter shows a kind of saw tooth behavior with strong asymmetries between cold and warm period. Thus it seems that our theory cannot represent the real climate. Such a negative statement, however, is somehow misleading simply
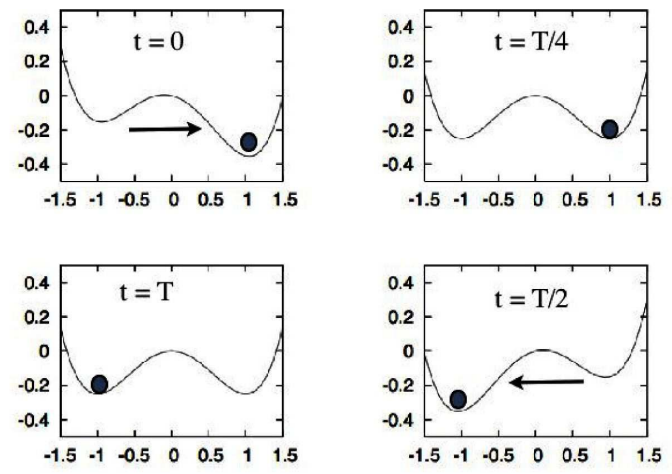

FIG. 4: A cartoon to explain the mechanism of stochastic resonance. The period of the oscillation is $T$. At time $t=0$ the climate states has low probability to jump from +1 to -1 . At time $t=T / 2$ the situation is reversed: the probability no to jump is extremely small. At time $t=T$ we start a new cycle.

because the model so far used, namely the energy balance model (6), includes only the simplest possible feedback due to radiation. More sophisticated effects, like the temperature-precipitation effect, can change the situation. Based on the above discussion we can make the following statement:

- stochastic resonance is able to show that the external "weak" forcing due to Milankovitch cycle can be amplified by internal non linear dynamics of the climate;

- climate dynamics shows a long time scale behavior due to non linear feedback;

- noise can develop long time scale behavior if there exists multiple equilibriums;

The most important feature coming out from our analysis is that it is crucial to understand the non linear interaction between fast a and slow time variables in climate models. Time scales of order 1000 years or more, do no rule out the importance of the dynamics of fast variables which usually characterize atmospheric and/or oceanic circulations'. This, I think, is an important feature even in more sophisticated and/or realistic climate models.

The mechanism of stochastic resonance has been recently invoked to explain the observed climatic variation on a shorter time scale with respect to the Milankovitch forcing. During the last 100, 000 years, the earth climate exhibits abrupt changes from relatively cold to warm states, different to those observed in the 100,000 year cycles. According to Alley et.al. 8], the climate changes occurred on time intervals which are multiple of 1,500 years, i.e. the time to switch from one climate state to the other is either 1,500 or 3,000 or 4,500 and so on. This rather peculiar behavior calls for an explanation which, 
not surprisingly, can be given in terms of stochastic resonance. As we previously discussed for equation (7), the effect of the periodic forcing is to decrease and increase (depending on the time $t$ ) the probability of a switching from a climate state to the other. With reference to figure (4), if at time $t=T / 2$ the system does not switch, then it takes a full period $T$ for the possibility to make a jump. This is certainly the case if the noise intensity is smaller than $\sigma_{R}$. It follows that the probability of the exit time is not peaked around $T / 2$ but it is quantized with maxima in $(2 n+1) T / 2, n=1,2, \ldots$. To show that our analysis is correct, in figure (6) we plot the probability distribution of the time to switch for $\sigma=0.8 \sigma_{R}$ (symbols) and for $\sigma=\sigma_{R}$ (continuous lines). The quantization effect is quite clear as predicted by our simple analysis.

It appears that the observation of Alley et.al. may be explained by the mechanism of stochastic resonance as it was pointed out by the same authors. Actually, a theoretical analysis of a simplified ocean/atmospheric model, due to Ganopolski and Rahmstorft [9] shows that the effect of a periodic forcing in freshwater input over the North Atlantic Ocean combined with a suitable stochastic forcing, produces a stochastic resonance in cold/warm climate changes as observed in real data. In the Ganopolski and Rahmstorft model, the two climate states, between which one has climate transitions, represent two different thermoaline circulation in the Norht Atlantic Ocean, which are responsable for sea ice growth and destroy.

It is unclear, at this stage, whether stochastic resonance is or is not the possible explanation of the Alley et.al. data and more research is needed to be done for a reasonable assessment. At any rate, it is important to remark that abrupt climate changes as those observed during the Milankovitch cycle or over the last 100,000 years are hard to be explained without the idea of transition between multiple equilibriums triggered by the noise, which is the basic feature of stochastic resonance.

\section{STOCHASTIC RESONANCE IN COMPLEX SYSTEMS}

In the last ten to fifteen years the mechanism of stochastic resonance has been widely applied to a number of different physical and biological systems. Among them, the most striking applications concern neural systems pioneered by Moss and collaborator [4]. In most cases, experimental results show a remarkable agreement with respect to the qualitative and quantitative picture represented in figure (6). Also, in many applications one does not know the "equation of motions" for the system and, actually, periodic perturbation leading to a stochastic resonance are used as a "measure" of non trivial co-

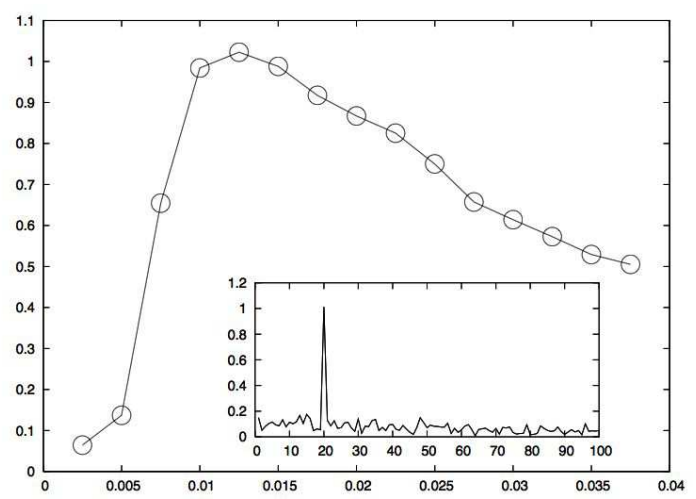

FIG. 5: The figure shows the Fourier amplitude $\left|F_{X}(\nu=\omega)\right|^{2}$ for the solution of equation (7) for different values of the noise amplitude $\sigma$. In the insert we show $\left|F_{X}(\nu)\right|^{2}$ for $\sigma=\sigma_{R}$, i.e.the optimal noise for which the Fourier amplitude at $\nu=\omega$ is maximum.

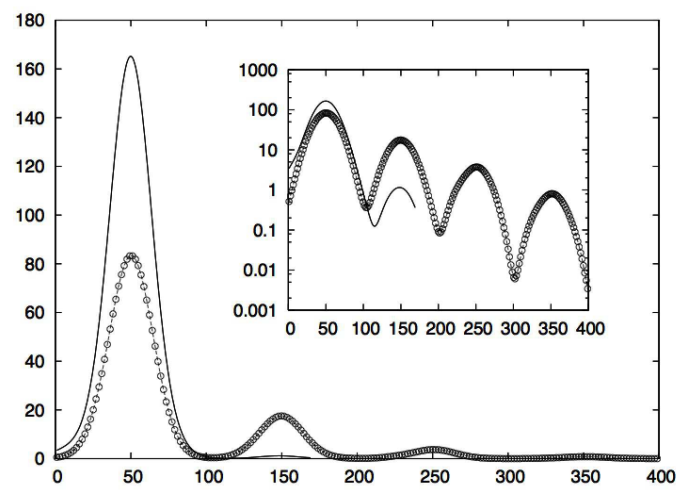

FIG. 6: Probability distribution of the random switching time $\tau$ for two different noise amplitude obtained as the solution of equation (7). The amplitude and the period of the forcing is the same. Line refers to $\sigma_{R}$ (see figure (5) while symbols refer to $\sigma=0.8 \sigma_{R}$. In the insert we show the same figure in log-lin plot. It is evident that for $\sigma$ smaller than $\sigma_{R}$ the probability distribution of $\tau$ is "quantized" at integer values of $(2 n+1) T / 2$.

operative phenomenon in the system under study (this is certainly the case for most neurophysiological systems).

When dealing with complex systems, one can find non trivial behavior of the stochastic resonance. In this section I show that this is the case for a network system composed by some "node' $\psi_{i}, i=1, \ldots N$, see figure (7).

Just to simplify the discussion, I assume that the dynamics of each node is controlled by the same equation and that the connectivity of the system is described by the matrix $L_{i j}$ :

$$
\frac{d \psi_{i}}{d t}=m \psi_{i}-g \psi_{i}^{3}+L_{i j} \psi_{j}+\sqrt{\sigma} \eta_{i}
$$

where $m>0$ and $g$ are real constant. Also, I will assume that $\sum_{i} L_{i j} \psi_{j} \equiv 0$ which clearly can be done without 


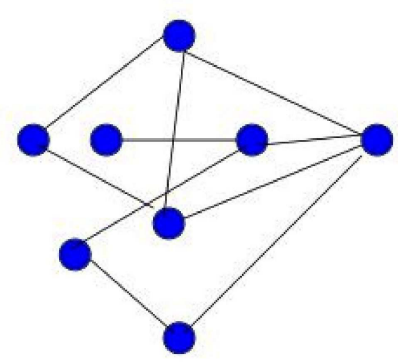

FIG. 7: A cartoon of our "complex" system. Each node satisfies equation (15). Lines describes the connectivity matrix $L_{i j}$.

lack of generality, and finally, that $\sum_{i} L_{i, j} \psi_{i} \psi_{j}>0$, i.e. the connectivity matrix does not introduce any "instability" in the dynamics of the system. The noise $\eta_{i}$ is $\delta$-correlated in time and $\left\langle\eta_{i} \eta_{j}\right\rangle=\delta_{i j}$.

The complexity of our problem, so to speak, is introduced by the matrix $L_{i j}$. We want now to study the behavior of (15) when an external periodic forcing is added to the system, i.e.

$$
\frac{d \psi_{i}}{d t}=m \psi_{i}-g \psi_{i}^{3}+L_{i j} \psi_{j}+\sqrt{\sigma} \eta_{i}+A_{i} \cos (2 \omega t)
$$

In order to simplify our work, let us focus on the "average" variable $\Phi=N^{-1} \sum_{i} \psi_{i}$. One should expect that for long enough period $T=2 \pi / \omega$ and a suitable noise $\sigma_{R}(m, g)$ a stochastic resonance can be observed. Note that I have defined $\sigma_{R}$ as an explicit function of the variables $m$ and $g$. By averaging (15) we obtain

$$
\frac{d \Phi}{d t}=m \Phi-g\left\langle\psi_{i}^{3}\right\rangle+\sqrt{\epsilon} \eta
$$

where $\sqrt{\epsilon} \equiv N^{-1} \sum_{i} \sqrt{\sigma}$ and $\langle\ldots\rangle \equiv N^{-1} \sum_{i} \ldots$. I assume that $\epsilon$ is independent of $N$, i.e. $\sigma$ is chosen in such a way that $\epsilon$ is a fixed quantity. The difficulty is to compute the term $\left\langle\psi_{i}^{3}\right\rangle$. For this purpose, we define $\phi_{i}$ such that $\psi_{i}=\Phi+\phi_{i}$, i.e. $\phi_{i}$ are "deviation" of $\psi_{i}$ from $\Phi$. Then we have $\left\langle\psi_{i}^{3}\right\rangle=\Phi^{3}+3 g \Phi\left\langle\phi_{i}^{2}\right\rangle$. This expression is correct as far as we can neglect the term $\left\langle\phi_{i}^{3}\right\rangle$ which, in most cases, is a good first approximation. Putting all together, we obtain:

$$
\frac{d \Phi}{d t}=\left(m-3 g\left\langle\phi_{i}^{2}\right\rangle\right) \Phi-g \Phi^{3}+\sqrt{\epsilon} \eta
$$

As we can see, the effect of complexity, i.e. the connectivity matrix $L_{i j}$, introduces a change in the linear term which now become a time dependent function. It
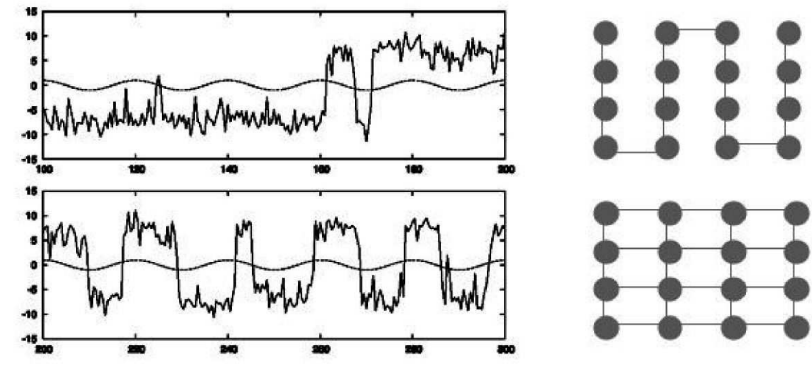

FIG. 8: Numerical simulation of equation (15) with the same parameters $m, g, \sigma$ and two different topology (shown in the figure): one dimensional lattice (upper panel), two dimensional lattice (lower panel). The amplitude and the period of the periodic forcing is the same for both cases. While the two dimensional lattice shows stochastic resonance, the same is not true for the one dimensional case.

can happen that $\left(m-3 g\left\langle\phi_{i}^{2}\right\rangle\right) \leq 0$ and if this is the case, transition between the two states (whatever they are) occur with a mechanism completely different with respect to what we discussed in the previous section. Is this the case? Everything depends on the quantity $\left\langle\phi_{i}^{2}\right\rangle$ and therefore on the connectivity matrix $L_{i j}$.

In order to make progress, let me first discuss what are the "statistical stable" equilibriums of (18). Let us define

$$
\Phi_{0}^{2} \equiv \frac{m-3 g\left\langle\phi_{i}^{2}\right\rangle}{g}
$$

We should expect that the statistical stable equilibriums are $\pm \Phi_{0}$. Then fluctuations around the equilibrium, which in first approximation we can describe as $\phi_{i}$, satisfy the equation:

$$
\frac{d \phi_{i}}{d t}=-\alpha \phi_{i}+L_{i j} \phi_{j}+\sqrt{\sigma} \eta_{i}
$$

where

$$
\alpha \equiv\left(m-3 g\left\langle\phi_{i}^{2}\right\rangle\right)-3 g \Phi_{0}^{2}=-2\left(m-3 g\left\langle\phi_{i}^{2}\right\rangle\right)
$$

Using (20) we can now estimate $\left\langle\phi_{i}^{2}\right\rangle$. Let us define $-\lambda_{n}$ the eigenvalues of $L_{i j}$. Then, by using a well known result in the theory of stochastic differential equation, we obtain:

$$
\left\langle\phi_{i}^{2}\right\rangle=\sum_{i} \phi_{i}^{2}=\frac{1}{2} \sum_{n} \frac{\sigma}{\alpha+\lambda_{n}}
$$

In the limit of large $N$, we can define the density of states $\rho(\lambda)$ with the approximation:

$$
\sum_{n} \rightarrow \int d \lambda \rho(\lambda)
$$


Putting everything together, we finally have:

$$
\left\langle\phi_{i}^{2}\right\rangle=\frac{1}{2} \int d \lambda \frac{\rho(\lambda)}{\alpha+\lambda_{n}}=\frac{1}{2} \int d \lambda \frac{\rho(\lambda)}{2\left(m-3 g\left\langle\phi_{i}^{2}\right\rangle\right)+\lambda}
$$

Equation (24) is a non linear equation relating the value of $\left\langle\phi_{i}^{2}\right\rangle$ to the density of states $\rho(\lambda)$,i.e. to the connectivity matrix $L_{i j}$. Once we have $\left\langle\phi_{i}^{2}\right\rangle$ we can comput $\Phi_{0}$. The tricky part of our problem is that we have computed $\left\langle\phi_{i}^{2}\right\rangle$ when $\langle A\rangle=0$, i.e. with no external forcing. If we now have an external forcing, all our computation for $\Phi_{0}$ and $\alpha$ should change taking into account $A$. One can compute perturbatively the effect in power of $A$ and take the first order for small $A$. The computation are done for a special case in [10]. The final results is that everything goes as in the theory discussed in section 2 but with a renormalized value of $A$,i.e.

$$
A_{R} \equiv A\left(1+\frac{3 g D}{1-2 D g}\right)
$$

where

$$
D \equiv \sigma \int d \lambda \frac{\rho(\lambda)}{\left(\alpha_{0}+\lambda\right)^{2}}
$$

and $\alpha_{0}$ corresponds (21) for $A=0$. For any practical purpose our result means the following. Let us imagine a network described by equation (15). Then the effect of a periodic forcing with amplitude $A$ on the average $\Phi$ is equivalent to a one dimensional problem (similar to (6) with a renormalized amplitude (25). The connectivity matrix fixes the value of the renormalization by (26). Thus, depending on $L_{i j}$, the effect of stochastic resonance can be enhanced or depressed. In figure (8) we demonstrate our result in a simple case. We consider the same system (i.e. same value of $m, g, A$ and $\sigma$ ) for connectivity matrix topologically equivalent to a one dimensional lattice (upper panel), and a bidimensional lattice. In the latter case the system shows stochastic resonance while the same is not true for the former case.

One main conclusion that we can outline from our discussion is particular relevant for climate theory. The physical meaning effect of an external forcing can be drastically different depending on the feedback in the system (in our case the matrix $L_{i j}$ ). There are cases, as we have seen, for which a small forcing or a small noise can trigger transitions and a naive computation of the relevant quantities (i.e. neglecting the effect discussed in this section) can lead us to wrong results. I want to argue that the above conclusions is relevant for other physical and biological systems.

\section{BROWNIAN MOTORS AND STOCHASTIC RESONANCE}

Up to now we have discussed the case of stochastic resonance in a more or less traditional fashion, i.e. when
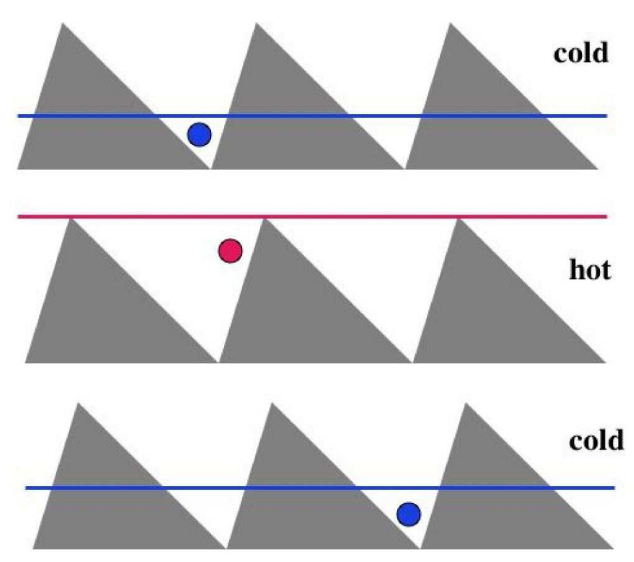

FIG. 9: A simple model of Brownian motor.Going from the top to the bottom of when the temperature is low (cold) a particle moving in the ratchet potential is trapped in one of the minima. Rising the temperature, the particle becomes almost free to move and, because its initial condition (minimum) is close the maximum on its right, the particle has more probability to go towards the right minimum. If the temperature is now decreasing the particle has more probability to be trapped in the right minimum with respect to be trapped in the minimum on the left

multiple equilibriums exist. We want now to understand whether there are other physical problems where a stochastic-like resonance can be of interest. One possible candidate, that I discuss in this section, is the case of Brownian motors.

The very idea of Brownian motors goes back to Feynman [11] and it can be summarized by saying that we want to use the energy of the thermal noise in order to make work. This can be done, consistently with the law of thermodynamics, if one considers a ratchet potential with a periodic time behavior of the temperature, see figure (9). Going from the top to the bottom of figure (9), we see that when the temperature is low (cold) a particle moving in the ratchet potential is trapped in one of the minima. Rising the temperature, the particle becomes almost free to move and, because its initial condition (minimum) is close the maximum on its right, the particle has more probability to go towards the minimum on the right. If the temperature is now decreasing the particle has more probability to be trapped in the right minimum with respect to be trapped in the minimum on the left. The overall picture is that of a diffusion process with more probability to move on the right than on the left. Thus, under the combined action of periodic temperature variation and the ratchet potential, we can have a non zero drift average velocity of the particle on the right direction: we have been able to extract work from the temperature field.

There are many kind of Brownian motors [12], the one shown in figure (9) is just an example. I will propose 


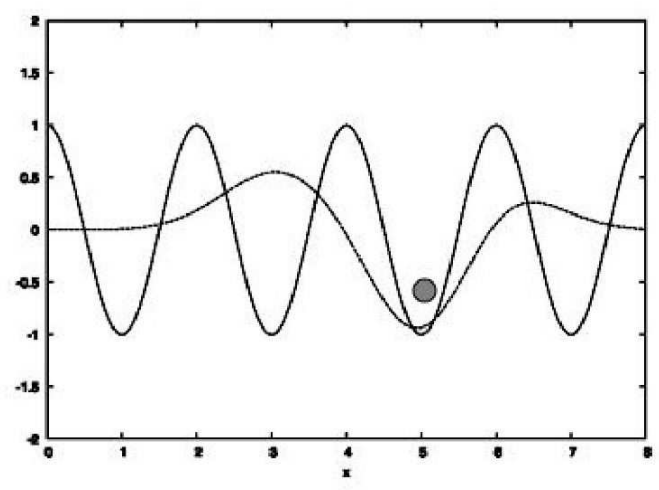

FIG. 10: The Brownian motor proposed in section 4. The particle moves in a periodic potential and noise is acting on it. A perturbing potential $\Phi(x-v t)$ localized in space is traveling in the system.

here a new kind of motors using the very same idea of stochastic resonance to produce a non zero current using the noise. Instead of a ratchet potential, let me consider a particle which feel a space periodic potential of period $L$. In the limit of overdamped friction, the equation of motion of the particle position $x$ is:

$$
\frac{d x}{d t}=-\frac{\partial V}{\partial x}+\sqrt{\sigma} \eta(t) \quad V(x)=V_{0} \cos (2 \pi x / L)
$$

We now introduce a time dependent forcing $F(x-v t)$ in the form of a moving localized perturbation as shown in figure (10). Actually we can thing of $F(\xi)$ as the effect of a moving potential perturbation, i.e.

$$
F(x-v t)=-\frac{\partial \Phi(x-v t)}{\partial x}
$$

Without the effect of $F$, the particle performs a diffusive process among the minima of $V$. The characteristic time $\tau_{L}$ to jump on the right or on the left can be computed by using the same method of section 2 . The action of the localized potential can introduce a new feature which is illustrated in figure (11).

If the speed $v$ of the perturbing potential $\Phi$ is properly chosen, then the particle has the possibility to jump on the right but not on the left when the perturbing potential $\Phi$ is in a given position. Then the particle remains trapped in the new minimum up to the time when the potential is now shifted to the next minimum and so on. In other words, the particle remains trapped in the moving potential $\Phi$ and it moves "balistically" at the same speed $v$ of the perturbing potential. In figure (12) I show $x(t)$ with and without $\Phi$ for a properly chosen value of $\sigma$. The initial particle position is $300 L$ and the initial perturbing potential is localized in $x=0$.

When $\Phi$ is close to $x=300$, the particle remains trapped and it moves with the speed $v$ for a rather long

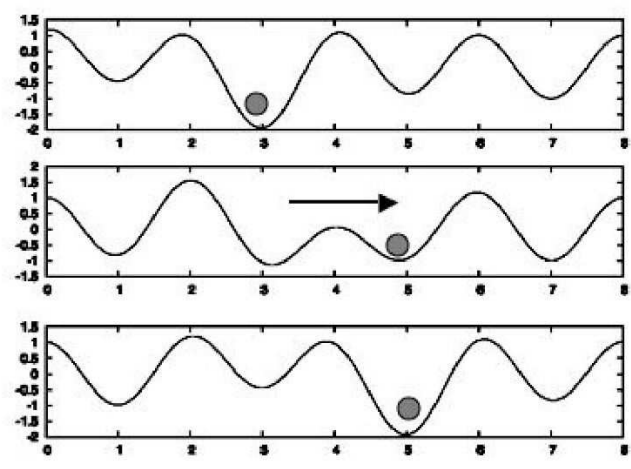

FIG. 11: The effect of the perturbing potential $\Phi(x-v t)$. From the top to the bottom, the particle can flip only in the positive $\mathrm{x}$-direction as the potential travels. The flip occurs only for a suitable combination of $v$ and the noise amplitude $\sigma$.

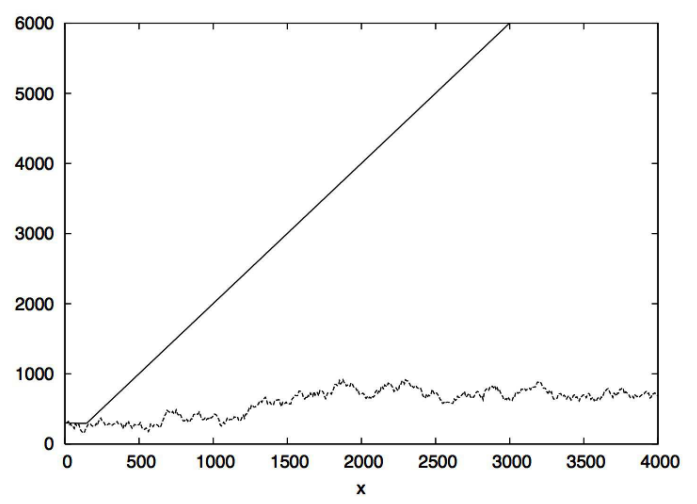

FIG. 12: The figure shows $x(t)$ with (straight line) and without (dotted line) $\Phi(x-v t)$ for a properly chosen value of $\sigma$. The initial particle position is $300 L$ and the initial perturbing potential is localized in $x=0$.

distance. According to our knowledge of stochastic differential equation, at each step the particle is following the potential with a probability close to 1 but not exactly equal to 1 . It follows that the particle is trapped in the potential $\Phi$ for a distance $D_{x}$ which is a random variable (it is the analogous of the random time $\tau_{L}$ discussed in detail in section 2).

As for the stochastic resonance, if the noise is to small or too large, $\Phi$ has no effect on the particle. The same is true if the velocity is too small or too large. We can check these qualitative statements in the following way. We consider an ensemble of $N$ particles and we study the average distance $\left\langle D_{x}\right\rangle$ as a function of noise variance $\sigma$ and the non dimensional velocity $v \tau_{L} / L$. This is done in figures (13) and (14) respectively.

In both cases, the effect of a resonant like phenomena is quite clear. Only for properly chosen values of $\sigma$ and $v$ we obtain the maximum of $\left\langle D_{x}\right\rangle$. Some recent experimental 


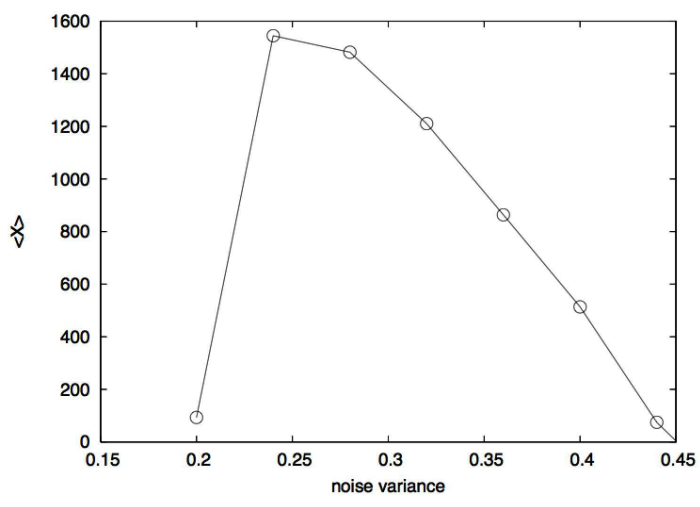

FIG. 13: The quantity $\left\langle D_{x}\right\rangle$ as a function of $\sigma$

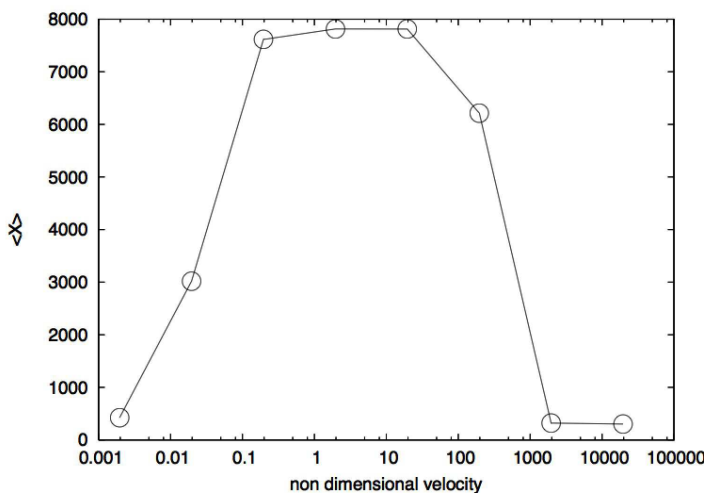

FIG. 14: The quantity $\left\langle D_{x}\right\rangle$ as a function of $v$

results [13] seem to confirm the numerical analysis done in this section.

\section{CONCLUSIONS}

In the last 30 years the scientific community did learn many different features on complex systems, starting by the pioneering works of Lorenz, Ruelle, Mandelbrot and others. In many cases, new ideas and tools have been introduced in order to "measure" complexity in an appropriate way. These tools can be used to reveal different features of underlying physical or biological mechanisms. In some sense, stochastic resonance is also a tool because it allows us to understand whether or not non linear effects can act in a cooperative way with the complex and chaotic behavior of a given system. On the other hand stochastic resonance is a mechanism in the full meaning of the word because it allows to get large effect from a small amplitude perturbation. There have been and still there are many applications of stochastic resonance in problems dealing with the amplification of signal to noise ratio, a quite traditional engineering problem.

In this paper I have reviewed some known and less known features of stochastic resonance. Some simple conclusions can be made.
- Stochastic resonance is counter intuitive phenomenon: it is not trivial that adding noise to a system we can enhance the deterministic periodic behavior.

- Stochastic resonance is a robust mechanism observed in many physical and biological systems. The notion of stochastic resonance is now cross disciplinary and new applications are found every year.

- We learn a lot in applying stochastic resonance in the theory of climatic change. As I mentioned in section 2 , it is a crucial step to understand that fast variables cannot be simply ignored in the study of long term climatic change, which is, overall, the basic idea introduced by stochastic resonance.

Acknowledgments I would like to thank many friends and colleagues for their help in early days of my research in stochastic resonance. Among them a special mention must be given to Giorgio Parisi, Alfonso Sutera and Angelo Vulpiani. Also, I am indebted with Micheal Ghil for his help and contribution. This paper represents the talk I gave on april 2006 for the L.F. Richardson lecture at EGU annual meeting in Vienna. I would like to thank the scientific committee of the EGU Richardson' medal and in particular D. Schertzer for his help and assistance. The paper has been written during a period of stage at the University of Chicago, Flash Center. I thank L. Kadanoff, D. Lamb, B. Fischer for their kind invitation and support.

[1] R. Benzi, G. Parisi, A. Sutera, A. Vulpiani,Tellus, 34, 10, (1982)

[2] C. Nicolis, G. Nicolis, Tellus, 34, 22, (1982)

[3] R. Benzi, A. Sutera, A. Vulpiani, J. Phys. A, 14, L453, (1981)

[4] F. Moss, K. Wiesenfeld, Sci. Am., 273, 50, (1995)

[5] McNamara B, K. Wiesenfeld, Phys. Rev. A., 39, 4854, (1989)

[6] L. Giammaitoni, P. Hanggi, P. Jung, F. Marchesoni, Rev. Mod. Phys., 70, 223, (1996)

[7] R. Benzi, Phys. Rev. Lett., 95, 024502, (2005)

[8] R.B. Alley, S. Anandankrishnan, P. Jung, Paleoceanography, 16, 190, (2001)

[9] A. Ganopolski, S. Rahmstorf, Phys. Rev. Lett., 88, 3, 038501-1, (2002)

[10] R. Benzi, A. Sutera, J. Phys. A, 37, L391, (2004)

[11] R. P. Feynman, R.B. Leighton, M. Sands, The Feynman Lectures on Physics, Vol. 1, Chap. 46, Addison Wesley, Reading MA (1963)

[12] P. Riemann, P. Hanggi, Appl. Phys. A, 75, 169 (2002)

[13] M. Schiavoni, F.-R. Carminati, L. Sanchez-Palencia, F. Renzoni, G. Grynberg, Europhy. Lett., 59, 493, (2002) 